\title{
Direct numerical simulations of turbulent channel flow with transverse square bars on one wall
}

\author{
By S. LEONARDI ${ }^{1}$, P. ORLANDI ${ }^{1}$, R. J. SMALLEY ${ }^{2}$, \\ L. DJENIDI ${ }^{3}$ AND R. A. ANTONIA ${ }^{3}$ \\ ${ }^{1}$ Dipartimento di Meccanica e Aeronautica, Università Degli Studi di Roma "La Sapienza", \\ 00184, Rome, Italy \\ ${ }^{2}$ Energy and Resources Research Institute, University of Leeds, Leeds LS2 9JT, UK \\ ${ }^{3}$ Discipline of Mechanical Engineering, University of Newcastle, NSW 2308, Australia
}

(Received 15 May 2003 and in revised form 15 June 2003)

Direct numerical simulations have been carried out for a fully developed turbulent channel flow with a smooth upper wall and a lower wall consisting of square bars separated by a rectangular cavity. A wide range of $w / k$, the cavity width to roughness height ratio, was considered. For $w / k \geqslant 7$, recirculation zones occur immediately upstream and downstream of each element while mean streamlines and spatial distributions of the skin frictional drag indicate that each element is virtually isolated. The maximum form drag occurs at $w / k=7$ and coincides with the minimum skin frictional drag. The dependence on $w / k$ of the Clauser roughness function reflects that of the form drag.

\section{Introduction}

Turbulent flows over rough surfaces are often encountered in practice; in the atmosphere, the underlying surface is usually rough, while, in an engineering context, pipes and ducts cannot be regarded as hydraulically smooth, especially at high Reynolds numbers. Rough surfaces may be used to enhance heat transfer, albeit at the expense of increasing the drag; alternatively, the roughness geometry may be selected so as to decrease the drag, e.g. by using riblets, or delay transition. Since the roughness can seriously degrade the performance of airfoils, wings and turbomachinery blades, the ability to predict its effect is important. According to the classical scheme (e.g. Nikuradse 1933; Clauser 1954; Perry, Schofield \& Joubert 1969; Raupach, Antonia \& Rajagopalan 1991), the rough-wall boundary condition affects the mean velocity distribution in the inner region (in the case of a channel flow, $y / h \lesssim 0.2$, where $h$ is the half-width of the channel). The effect of the roughness is to shift the mean velocity profile, with respect to that on a smooth wall, by an increment $\Delta U^{+}$, referred to as the roughness function, i.e.

$$
U^{+}=\kappa^{-1} \ln y^{+}+C-\Delta U^{+},
$$

where $C$ and $\kappa$ are constants and + denotes normalization by either $U_{\tau}\left(\equiv(\tau / \rho)^{1 / 2}\right)$, $\tau$ is the wall shear equal to the sum of the viscous (or skin frictional) drag and the form drag or $v / U_{\tau}$. The roughness function $\Delta U^{+}$depends on the density (defined as the total roughness frontal area per unit wall area), height $(k)$ and nature of the 
roughness. Because of the general difficulties in making reliable measurements in the vicinity of the roughness elements, $\tau$ is usually inferred by assuming the validity of (1.1). Direct numerical simulations can provide an accurate determination of $\tau$, albeit at small Reynolds numbers. They also allow quantification of the relative contributions to $\tau$ of the form and skin frictional drag. In this paper, we examine in detail how these contributions depend on $w / k$, after identifying and describing the flow regimes associated with particular values of $w / k$. We also estimate $\Delta U^{+}$ and its dependence on $w / k$. Most of the results presented here are for $k / h=0.2$ and $R e=4200$, where $R e=\left(U_{c} h / v\right)$ is the Reynolds number, $U_{c}$ is the centreline velocity and $v$ is the kinematic viscosity. A few simulations were carried out for $k / h$ as small as 0.1 and $R e$ as large as 10400 to assess the effect of these two parameters on the results.

\section{Numerical procedure}

The incompressible non-dimensional Navier-Stokes and continuity equations may be written as

$$
\frac{\partial U_{i}}{\partial t}+\frac{\partial U_{i} U_{j}}{\partial x_{j}}=-\frac{\partial P}{\partial x_{i}}+\frac{1}{R e} \frac{\partial^{2} U_{i}}{\partial x_{j}^{2}}+\Pi, \quad \nabla \cdot \boldsymbol{U}=0,
$$

where $\Pi$ is the pressure gradient required to maintain a constant flow rate; $U_{i}$, the component of the velocity vector in the $i$-direction and $P$ the pressure. All the quantities have been normalized by a combination of $U_{c}$ and $h$. The NavierStokes equations have been discretized in an orthogonal coordinate system using the staggered central second-order finite-difference approximation. Here we recall only the main features since details of the numerical method can be found in Orlandi (2000). The discretized system is advanced in time using a fractional-step method with viscous terms treated implicitly and convective terms explicitly. The large sparse matrix resulting from the implicit terms is inverted by an approximate factorization technique. At each time step, the momentum equations are advanced with the pressure at the previous step, yielding an intermediate non-solenoidal velocity field. A scalar quantity $\Phi$ projects the non-solenoidal field onto a solenoidal one. A hybrid lowstorage third-order Runge-Kutta scheme is used to advance the equations in time. The roughness is treated by the immersed boundary technique described in detail by Fadlun et al. (2000). This approach allows the solution of flows over complex geometries without the need of computational intensive body-fitted grids. It consists of imposing $U_{i}=0$ on the body surface, which does not necessarily coincide with the grid (• in figure 1). Another condition is required to avoid the geometry being described in a stepwise way. Fadlun et al. (2000) showed that second-order accuracy is achieved by evaluating the velocities at the closest point to the boundary $(x$ in figure 1) using a linear interpolation. This accounts for the physical behaviour of a linear mean velocity profile very near the boundary even for turbulent flows, albeit at the expense of clustering more points near the body. Fadlun et al. (2000) showed that the velocity goes to zero where the boundary of the body is supposed to be, even if this location does not coincide with the grid. The integration of $(2.1)$ requires $5 \%$ more CPU time with the immersed boundaries than without them.

\section{Flow configuration}

Direct numerical simulations have been performed for a fully developed turbulent channel flow with square bars on the bottom wall (figure 2). Several values of $w / k$ 


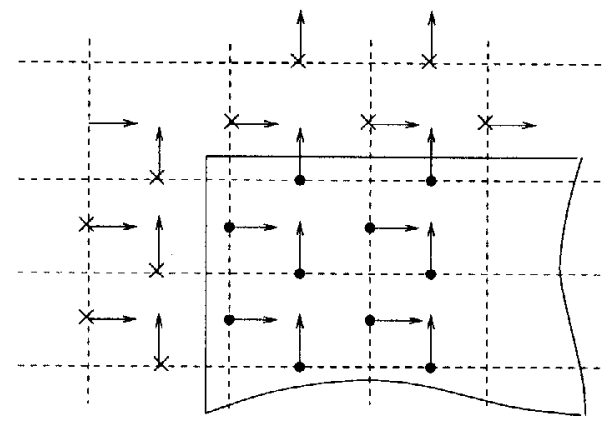

FIGURE 1. Geometrical sketch of immersed boundary method. Arrows, velocity vectors as defined in a staggered grid $(----)$; $\bullet$, points inside the roughness element; $\times$, points nearest to the boundary of the roughness element. The solid line delineates part of the roughness element.

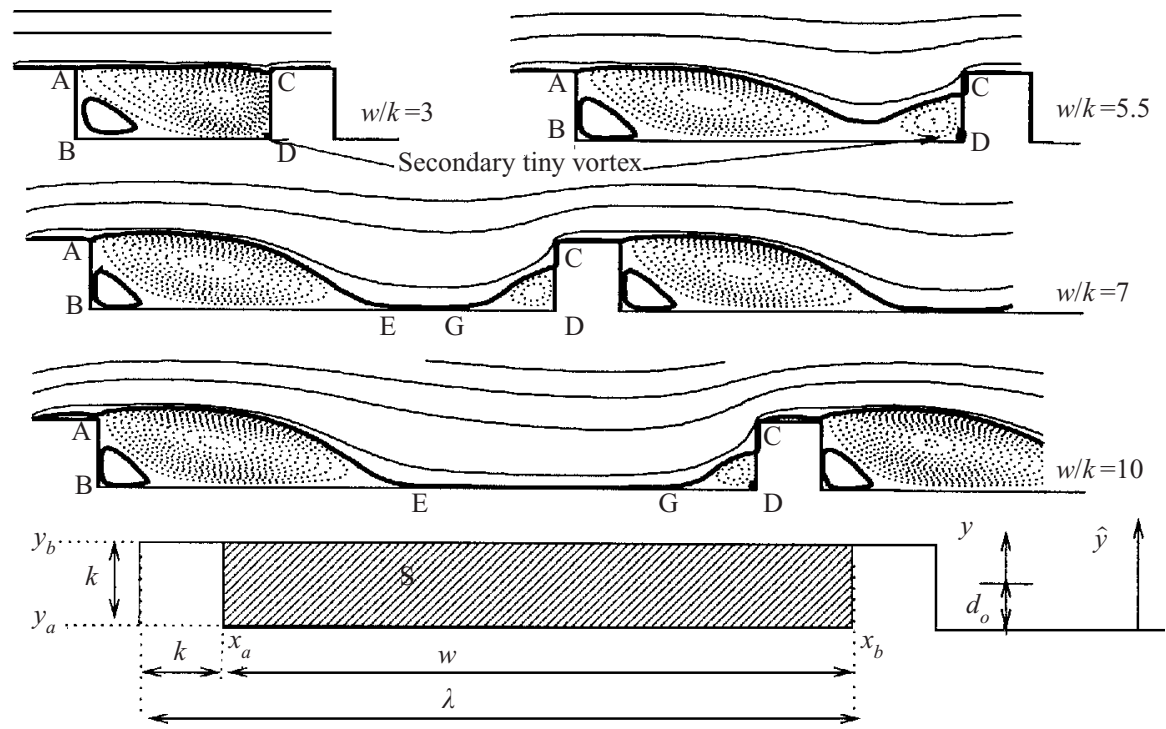

FIGURE 2. Mean streamlines, averaged with respect to time and $z$, for different $w / k$ and $k / h=0.2$. The thick line corresponds to the zero streamfunction; - , positive streamfunction, --- , negative (primary recirculation zone). The definitions of $k, w, d_{o}, y, \hat{y}$ and $\lambda$ are indicated. Flow is left to right.

have been investigated $(0.33,0.6,1,2.07,3,4,5.5,7,8,9,10,19)$ with $k=0.2 h$. Periodic boundary conditions apply in the streamwise $(x)$ and spanwise $(z)$ directions, and there is a no-slip condition at the wall in the wall-normal direction $(y)$. The computational box is $8 h \times 2 h \times \pi h$ in $x, y$ and $z$ respectively. The Reynolds number is $R e=4200$ and corresponds to $R e_{\tau}=180$ when both walls are smooth. The flow rate has been kept constant in all simulations. Hence, $U_{\tau}, k^{+}$and $R e_{\tau}$ depend on $w / k$. The value of $R e_{\tau}$ on the rough wall ranges between 190 (for $w / k=1$ ) and 460 (for $w / k=7$ ) and $k^{+}$ranges from about 40 to about 90 . For this particular surface (with an infinite aspect ratio), Bandyopadhyay (1987) found that, for $k^{+} \geqslant 10$, the flow can be assumed to be 'fully rough' so that the roughness function $\left(\Delta U^{+}\right)$depends only on $k^{+}$and not on the Reynolds number. We have verified this (the results are not given here) by running four simulations $(R e=4200, k=0.2 h ; R e=8400, k=0.1 h$ and 
$0.15 h ; R e=10400, k=0.1 h)$ for $w / k=3$. We have also verified that the results are not affected by blockage. Three different grids have been used for all the simulations at $R e=4200: 200 \times 140 \times 97,400 \times 140 \times 97$ and $300 \times 140 \times 97$. The first two are non-uniform in $y$ while the third is non-uniform in $x, y$. In the normal direction, the points are clustered near the wall: within the cavity $\left.\Delta y\right|_{\min }=0.005$. The mesh increases toward the channel centreline, with $\left.\Delta y\right|_{\max }=0.03$. In $x$, points are clustered near the roughness elements. Since results obtained with the $300 \times 140 \times 97$ and $400 \times 140 \times 97$ grids are virtually identical, only results for the latter grid are shown here. The simulations at $R e=8400$ and $R e=10400$ have been carried out with a grid $600 \times 177 \times 193$ on a cluster of PCs.

\section{Mean streamlines}

Prior to quantifying the magnitudes of the form and skin frictional drag over the rough wall, we investigated the effect of $w / k$ on the overall behaviour of the flow between roughness elements. Mean streamlines, averaged with respect to time and $z$ are shown in figure 2 . For $w / k \leqslant 4$, a separation occurs at the trailing edge of the element (point A) and reattachment is on the opposite vertical wall. The cavity is occupied by a large recirculation region with two secondary vortices, of opposite direction to the main recirculatory zone, in the corners (D) and (B). For $w / k \geqslant 7$, the flow reattaches on the bottom wall (point E) at about $4.8 k$ downstream of the back face of the element $(B E \simeq 4.8 k)$. The near-wall streamlines are essentially horizontal between $\mathrm{E}$ and $\mathrm{G}$, the distance GD being approximately $1.5 k$. As the next element is approached, the streamlines are tilted upward and separation occurs. This is in very close agreement with the flow visualizations of Liu, Kline \& Johnston (1966). They reported a reattachment length of $5 k$ for $w / k=7$ and $w / k=11$. Le, Moin \& Kim (1997) performed a DNS of a turbulent flow over a backward facing step with inlet and outlet boundary conditions; the streamwise length of the domain was $20 k$. They showed that the instantaneous reattachment location, averaged in $z$, oscillates between $X_{r} / k=4.8$ and $X_{r} / k=8\left(X_{r}\right.$ is measured from the step) with an averaged value of about $6.3 \mathrm{k}$. Liu et al. (1966) reported a reattachment length of $6 k$ for $w / k=23$. In the present case $\mathrm{BE}=5.8 k$ for $w / k=19$. The previous results suggest that, when $w / k$ is large enough, the reattachment length is not influenced by the presence of other elements, while for $w / k \simeq 7$, the vertical downstream wall (CD) causes an adverse pressure gradient (relative to a backward-facing step) which leads to a shorter reattachment length.

\section{Skin friction and form drag}

Figure 2 clearly shows that once $w / k$ exceeds a 'critical' value $(\simeq 7)$, the flow remains virtually unchanged around a roughness element. Despite the slight increase in the reattachment length, the only noticeable difference in the patterns between $w / k=7$ and 10 is in the length of the region where the streamlines are parallel to the bottom wall (EG). The size and strength of the recirculation zones do not change significantly for $w / k \geqslant 7$. This is further corroborated by the non-dimensional viscous shear stress at the wall, $\left\langle C_{f}\right\rangle=\left(\mu \partial\left\langle U^{*}\right\rangle / \partial y^{*}\right)\left(1 / \rho U_{c}^{2}\right)=\left(R e^{-1}\right)(\partial\langle U\rangle / \partial y)_{y=0}$, where * indicates dimensional units and \langle\rangle denotes averaging with respect to time and $z$.

In figure $3,\left\langle C_{f}\right\rangle$ is shown for $w / k=3,4,7,8,9,10$ along the horizontal wall, with $s=0$ at the leading edge of an element. Over the ranges $-1<s / k<0$ and $1<s / k<5.5$, the $\left\langle C_{f}\right\rangle$ values agree reasonably well with each other for $w / k \geqslant 7$. 


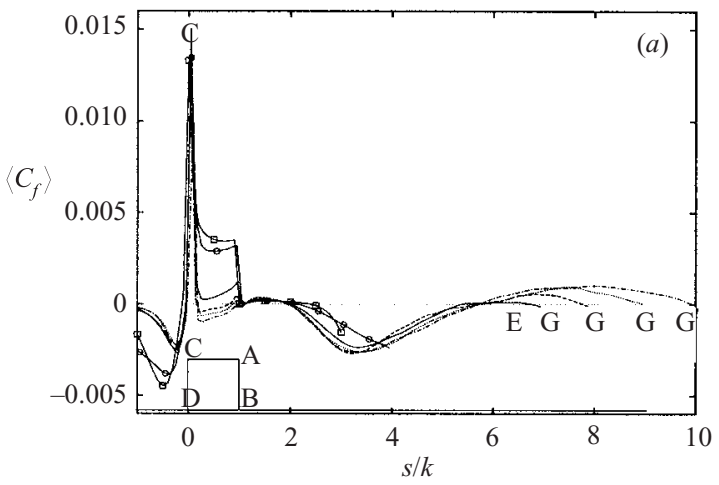

Figure 3. (a) $\left\langle C_{f}\right\rangle$ for different values of $w / k .-\square-, w / k=3 ;-\circ-, 4 ;-, 7$; ,$---- 8 ; \cdots . . ., 9 ;--\ldots, 10 .(b)$ Contours of pressure around a roughness element: - , positive, $\cdots$, negative. The interval between successive contour levels is 0.01 .
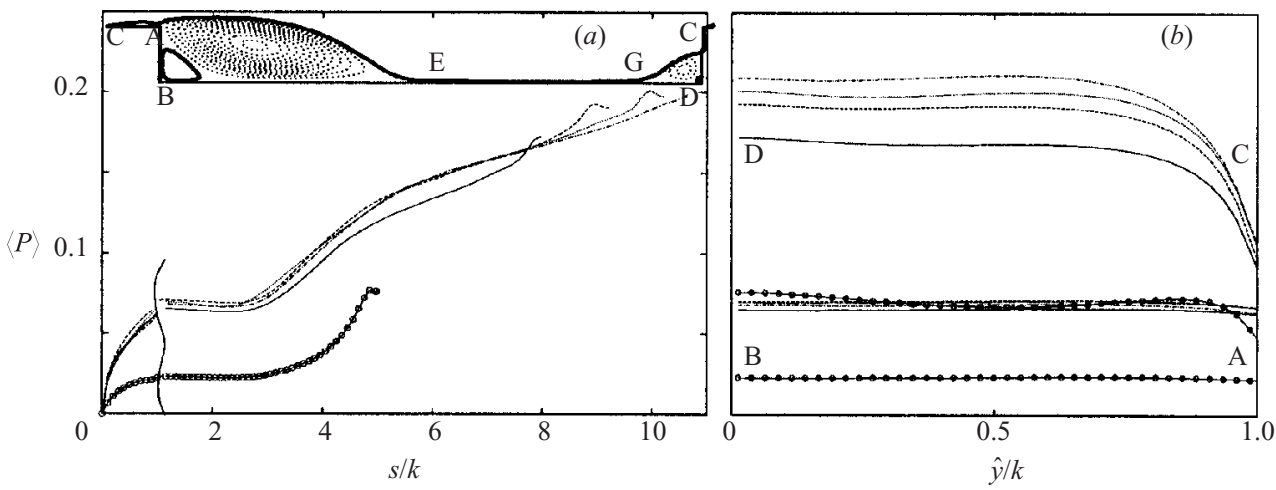

Figure 4. Distributions of the pressure $\langle P\rangle$ along the walls. (a) Horizontal walls (CA + BD), (b) vertical walls $(\mathrm{AB}+\mathrm{CD})$. Symbols as in figure 3. The inset near the top of $(a)$ shows streamlines for $w / k=10$. The thick line corresponds to the zero streamfunction; ----, negative streamfunction (primary recirculation zone).

Within the cavity, $\left\langle C_{f}\right\rangle$ is negative underneath the primary recirculation region, with one negative peak for $w / k \leqslant 4$ and two for $w / k \geqslant 7$. For $w / k \geqslant 7$, the distance EG, over which the mean viscous shear is positive, increases with $w / k$.

On the element crests $(0<s / k<1)$, the same peak is observed in each case, although separation occurs for $w / k \geqslant 7$. By increasing $w / k$, the intensity of the recirculation zone increases and $\left\langle C_{f}\right\rangle$ is reduced. This is due to the pressure field near the roughness element. Maximum and minimum values of $\langle P\rangle$ (figure 3) are located on the upstream face and crest of the element, so that a strong pressure gradient drives the flow outward around the vicinity of the leading edge of the element. By increasing $w / k$, the pressure gradient increases, resulting in separation above the crests for $w / k \geqslant 8$.

The pressure variation along both the horizontal and vertical wall surfaces is shown in figure 4 . It is assumed that for each roughness geometry, $\langle P\rangle$ is zero at $s / k=0$. On the horizontal wall $\mathrm{BD},\langle P\rangle$ is constant over a distance of about $2 k$, because the vortex at $\mathrm{B}$ is too weak to cause a significant pressure gradient. For a sparse roughness $(7 \lesssim w / k \lesssim 10),\langle P\rangle$ grows linearly with $x$, from approximately the centre of the vortex to the reattachment point $\mathrm{E}(4 \lesssim s / k \lesssim 6)$. The similarity between the different pressure distributions over this range of $w / k$ suggests that the roughness 

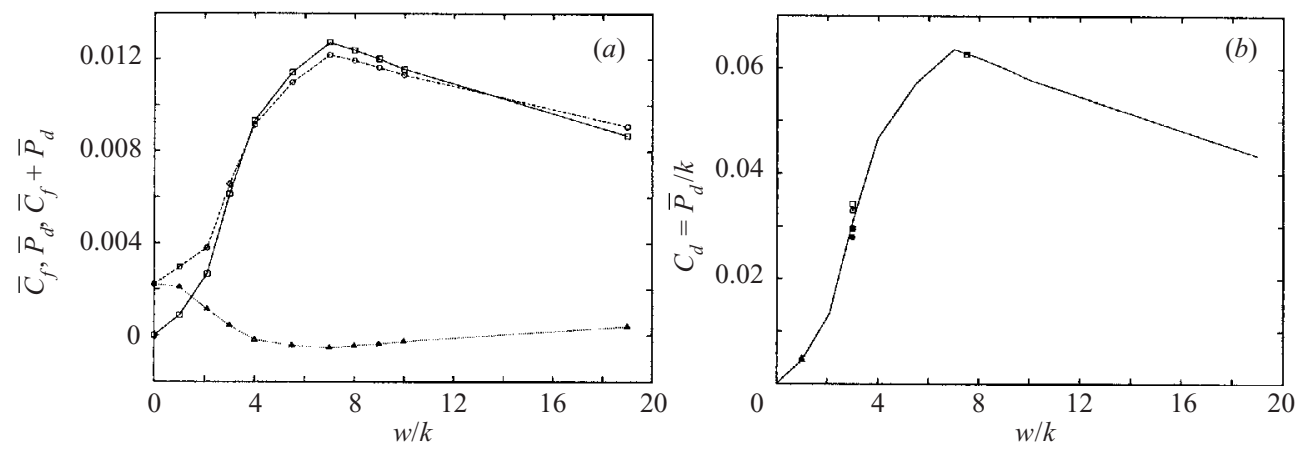

Figure 5. (a) Dependence of the skin frictional drag $\overline{C_{f}}$, form drag $\overline{P_{d}}$ and of their sum on $w / k$. Averaging is over one roughness wavelength. $\triangle, \overline{C_{f}} ; \square, \overline{P_{d}} ; \circ, \overline{P_{d}}+\overline{C_{f}} ; k / h=0.2$, $R e=4200$. (b) Drag coefficient $\overline{P_{d}} / k . \longrightarrow, k=0.2 h, R e=4200 ; \circ, k=0.1 h, R e=8400 ; \bullet$, $k=0.15 h, R e=8400 ; \triangle, k=0.15 h, R e=8400 ; \mathbf{\Delta}, k=0.15 h, R e=6300 ; \square, k=0.1 h, R e=10400$.

elements are isolated. Figure $4(b)$ shows $\langle P\rangle$ on the two vertical walls (the origin of $\hat{y}$ is on the cavity floor, figure 2 ). As a result of the recirculation region immediately downstream of the roughness element, $\langle P\rangle$ is constant on section AB. On the opposite wall $(\mathrm{CD}),\langle P\rangle$ is approximately constant in the separated region up to $0.75 \mathrm{k}$, and decreases near the leading edge $(C)$ of the element (see figure 3 ).

Over a streamwise wavelength $\lambda$, the relative contributions of $\left\langle C_{f}\right\rangle$ and $\left\langle P_{d}\right\rangle$ to $\tau$ are $\overline{C_{f}}=\lambda^{-1} \int_{0}^{\lambda}\left\langle C_{f}\right\rangle \mathrm{d} s$ and $\overline{P_{d}}=\lambda^{-1} \int_{0}^{\lambda}\langle P\rangle \boldsymbol{n} \cdot \boldsymbol{x} \mathrm{d} s$ respectively, where $\overline{P_{d}}$ is the form drag obtained by projecting $\langle P\rangle$ onto the $x$-direction ( $\boldsymbol{n}$ is the normal to the surface). For a square bar roughness, $P_{d}$ is simply the difference between the pressure integrals along each vertical wall. Figure 5(a) shows that there are two distinct regimes for both $\overline{C_{f}}$ and $\overline{P_{d}}$. For $w / k \lesssim 7, \overline{C_{f}}$ needs to decrease as $w / k$ increases. This corresponds to a lengthening of the separated region adjacent to the cavity floor. Beyond $w / k=7$, $\overline{C_{f}}$ increases slowly as EG increases. The geometry (with the shortest EG) that minimizes $\overline{C_{f}}$ corresponds to $w / k=7 . \overline{P_{d}}$ has a maximum at $w / k=7$ and exhibits a weak dependence on $w / k$ for $5<w / k<10$. This behaviour is in agreement with the experimental results of Furuya, Miyata \& Fujita (1976) who used cylindrical rods attached to the wall, transversely to the flow. This suggests that $w / k$ is the key parameter of the flow, whereas the shape of the roughness element is likely to be less important. The form drag per unit area $C_{d}=\overline{P_{d}} / k\left(=\int P^{*} \mathrm{~d} y^{*} \mathrm{~d} z^{*} /\left(\rho U^{* 2} \lambda^{*} k^{*}\right)\right)$, or drag coefficient, is shown in figure $5(b)$. The distribution for $k / h=0.2$ and $R e=4200$, as estimated from figure $5(a)$, represents the other results obtained at different $k / h$ and $R e$ to a good approximation. The implication of figure $5(b)$ is that, for this flow, $C_{d}$ depends only on $w / k$ and it is not sensitive to $k / h$ and $R e$.

To gain some insight into the relationship among $\overline{P_{d}}, \overline{C_{f}}$ and the Reynolds shear stress at the edge of the cavity, the Navier-Stokes equation for $U$ was integrated over the cavity region $\mathrm{S}$ (see figure 2) bounded by consecutive roughness elements $\left(x_{1}, x_{2}, x_{3}\right.$ are equivalent to $\left.x, y, z\right)$ :

$$
\int_{S}\left(\frac{\partial U}{\partial t}+\frac{\partial U U_{j}}{\partial x_{j}}\right) \mathrm{d} x_{1} \mathrm{~d} x_{2} \mathrm{~d} x_{3}=\int_{S}\left(-\frac{\partial P}{\partial x}+\frac{1}{R e} \frac{\partial^{2} U}{\partial x_{j}^{2}}\right) \mathrm{d} x_{1} \mathrm{~d} x_{2} \mathrm{~d} x_{2} .
$$

Since the flow rate is constant $(\partial Q / \partial t=0)$, and there is periodicity in $x$ and $z,(5.1)$ 


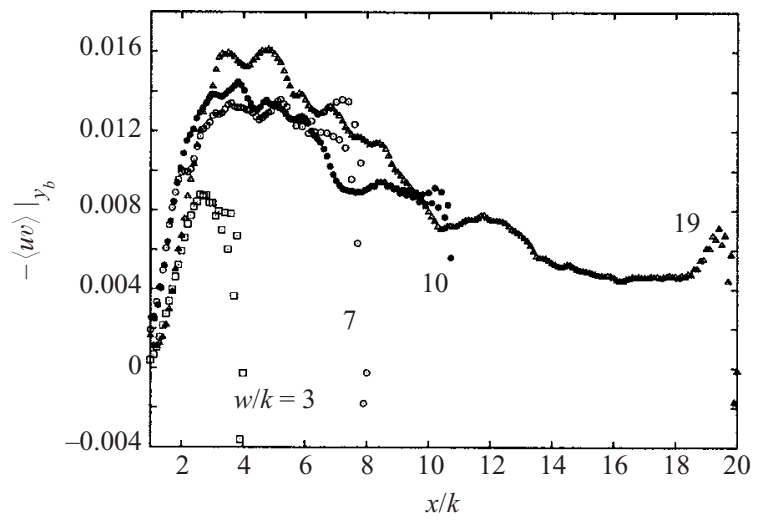

FIGURE 6. $-\left.\langle u v\rangle\right|_{y_{b}}$ as a function of $x / k . \square, w / k=3 ; \circ, w / k=7 ; \bullet, w / k=10 ; \triangle, w / k=19$. The range of $x$ on the abscissa extends from $x_{a}$ to $x_{b}$.
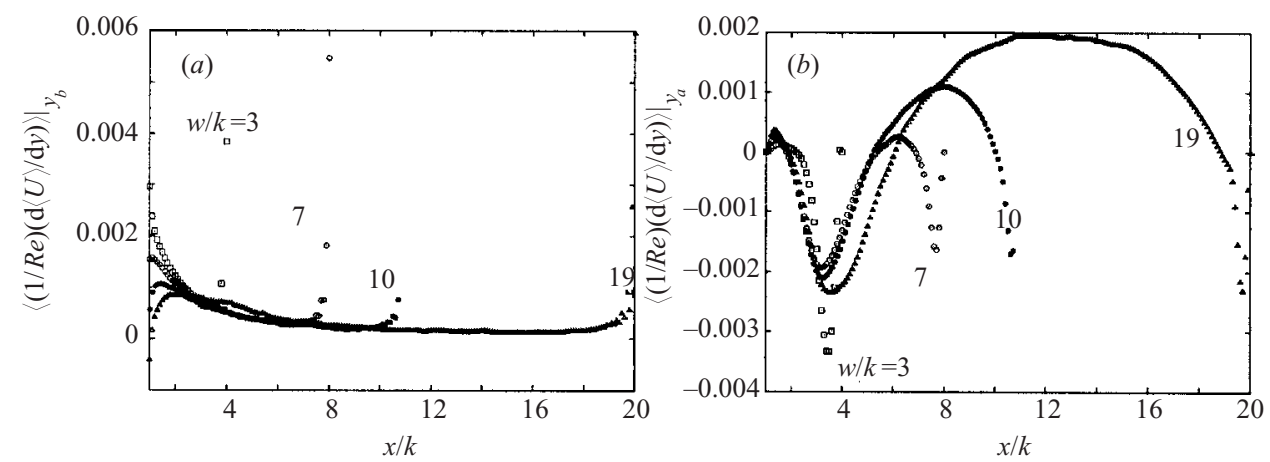

FIGURE 7. $\left.\langle(1 / R e)(\mathrm{d}\langle U\rangle / \mathrm{d} y)\rangle\right|_{y}$ as a function of $x / k$. (a) $y=y_{b}$ (plane of the crests), (b) $y=y_{a}$ (bottom wall). Symbols and range for $x$ are as in figure 6 .

can be simplified to

$\int_{y_{a}}^{y_{b}}\left\langle\left. P\right|_{x_{b}}\right\rangle-\left\langle\left. P\right|_{x_{a}}\right\rangle \mathrm{d} y=\left.\int_{x_{a}}^{x_{b}} \frac{1}{R e} \frac{\mathrm{d}\langle U\rangle}{\mathrm{d} y}\right|_{y_{b}} \mathrm{~d} x-\left.\int_{x_{a}}^{x_{b}}\langle u v\rangle\right|_{y_{b}} \mathrm{~d} x-\left.\int_{x_{a}}^{x_{b}} \frac{1}{R e} \frac{\mathrm{d}\langle U\rangle}{\mathrm{d} y}\right|_{y_{a}} \mathrm{~d} x$,

where $U=\bar{U}+u$. After dividing by $\lambda$, we obtain

$$
\overline{P_{d}}=\left.\frac{1}{\lambda} \int_{x_{a}}^{x_{b}} \frac{1}{R e} \frac{\mathrm{d}\langle U\rangle}{\mathrm{d} y}\right|_{y_{b}} \mathrm{~d} x-\left.\frac{1}{\lambda} \int_{x_{a}}^{x_{b}}\langle u v\rangle\right|_{y_{b}} \mathrm{~d} x-\left.\frac{1}{\lambda} \int_{x_{a}}^{x_{b}} \frac{1}{R e} \frac{\mathrm{d}\langle U\rangle}{\mathrm{d} y}\right|_{y_{a}} \mathrm{~d} x .
$$

Figure 6 shows that $\left.\langle u v\rangle\right|_{y_{b}}$ is maximum near the reattachment point. Hence, the integral of $\left.\langle u v\rangle\right|_{y_{b}}$ divided by $\lambda\left(\overline{P_{d}}\right.$ is the pressure drag divided by $\left.\lambda\right)$ is maximum when $w / k=7$. This is the smallest value of $w / k$ for which reattachment occurs on the bottom wall. Downstream of the reattachment point $(x / k \geqslant 8),\left.\langle u v\rangle\right|_{y_{b}}$ decreases and at a sufficiently large distance $(x / k \simeq 15)$ from the element, it approaches the value for a flat surface. For small values of $w / k$, reattachment occurs on the vertical downstream wall, and since the separated region is more stable, the magnitude of $\langle u v\rangle$ is smaller. The value of $(1 / R e) \mathrm{d}\langle U\rangle /\left.\mathrm{d} y\right|_{y_{b}}$ (figure $7 a$ ), decreases slowly with $x$ so that its integral with respect to $x$, after dividing by $\lambda$, decreases when $w / k$ increases.

The quantity $(1 / R e) \mathrm{d}\langle U\rangle /\left.\mathrm{d} y\right|_{y_{a}}$ (figure $\left.7 b\right)$, represents the friction on the bottom wall. It is negative almost everywhere for $w / k=7$ since reattachment on the 

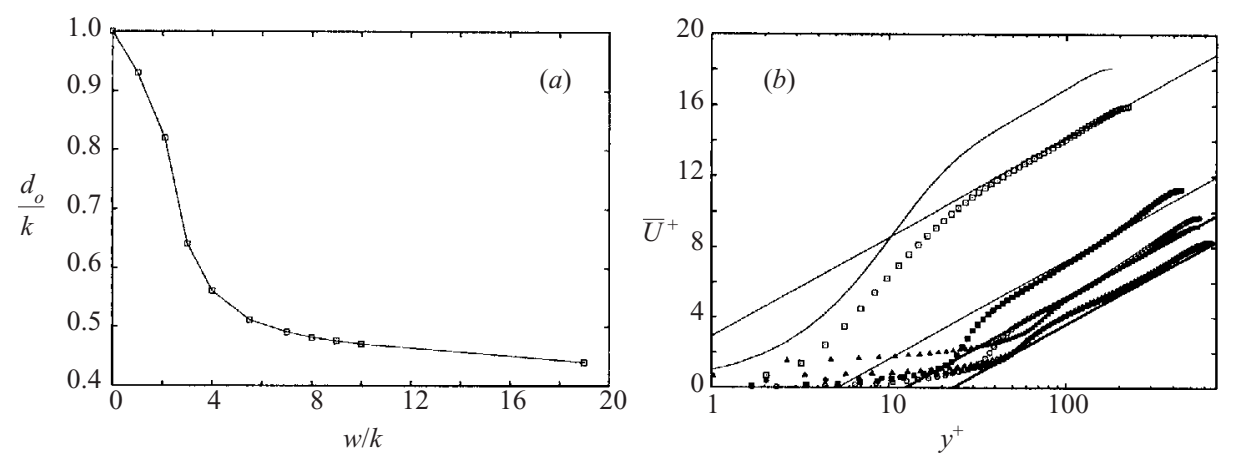

FIGURE 8. (a) Dependence of the displacement height $d_{o}$ on $w / k$. (b) Distributions of $\bar{U}^{+}$. Origin in $y$ estimated with the Jackson (1981) method. - Kim, Moin \& Moser (1987). $\square, w / k=1 ; \boldsymbol{\square}, 3 ; \circ, 4 ; \bullet, 7 ; \Delta, 9 ; \boldsymbol{\Delta}, 19$. The straight lines of reference are $\bar{U}^{+}=1 / 0.41 \ln y^{+}+C^{\prime}, C^{\prime}$ being $2.9,-4,-6.2,-7.7,-7.6,-6.1$ for $w / k=1,3,4,7,9,19$ respectively.

bottom wall is immediately followed by separation due to the downstream element. Collectively, the information in figures 6 and 7 implies that $P_{d}$ is likely to be maximum at $w / k \simeq 7$ since the contributions from the second and third terms on the right-hand side of (5.3) are maximum for that geometry. The contribution from the first term is negligible.

\section{Roughness function}

Since the results of the previous section allow $U_{\tau}$ to be estimated reliably, we now turn our attention to the estimation of $\Delta \bar{U}^{+}$and its dependence on $w / k$. There are three unknowns in (1.1): $U_{\tau}, \kappa$ and the origin for $y$. Usually, experimentalists determine $U_{\tau}$ and $d_{o}$ (measured from the base of the roughness element) by fitting the mean velocity data to (1.1) after assuming a value of $\kappa$ (usually 0.41 ). Perry $\&$ Joubert (1963) pointed out that several combinations of $U_{\tau}, d_{o}$ are possible so that there is inevitable ambiguity associated with their determination, even when the same type of roughness is considered. Since $U_{\tau}$ has been determined accurately here $\left(\equiv\left(\overline{P_{d}}+\overline{C_{f}}\right)^{1 / 2}\right)$, independently of $(1.1)$, only one unknown $\left(d_{o}\right)$ remains. We have followed two different approaches: that of Jackson (1981) who identified $d_{o}$ with the centroid of the moment of forces acting on the elements, and that in which $\kappa$ is assumed $(\simeq 0.41)$. The first approach is especially attractive since it allows $\Delta \bar{U}^{+}$to be estimated independently of (1.1). By defining $M$ as the moment due to the forces in the streamwise direction with respect the base of the element, $M=\int_{\mathrm{D}}^{\mathrm{C}}\langle P\rangle \hat{y} \mathrm{~d} s+$ $\int_{\mathrm{B}}^{\mathrm{A}}\langle P\rangle \hat{y} \mathrm{~d} s+\overline{C_{f}} k$ (see figure 2 for symbols), so that $d_{o}=M /\left(\overline{P_{d}}+\overline{C_{f}}\right)$. Figure $5(a)$ shows that when $w / k<2, \overline{P_{d}}$ is small so that $\overline{C_{f}} k$ provides the main contribution to $M$ and $d_{o} \sim k$, implying that the origin is virtually on the crest (figure $8 a$ ). As $w / k$ increases, the contribution from $\overline{P_{d}}$ dominates (figure $5 a$ ) and the moment due to $C_{f}$ can be neglected. For each of the limiting conditions, $(w / k \rightarrow \infty)$ and $(w / k \rightarrow 0), d_{o}$ approaches the expected limits of 0 and $k$ respectively. Figure $8(b)$ shows distributions of $\bar{U}^{+}$for a wide range of $w / k$ and the smooth channel flow distribution of Kim et al. (1987) is included as reference. Averaging has been carried out with respect to $x, z, t$ because, for a particular roughness density, there is no perceptible dependence on $s / k$ (position within one wavelength) for $\hat{y}>3 k$. As expected, the velocity exhibits a downward shift with respect to the smooth channel. The maximum 


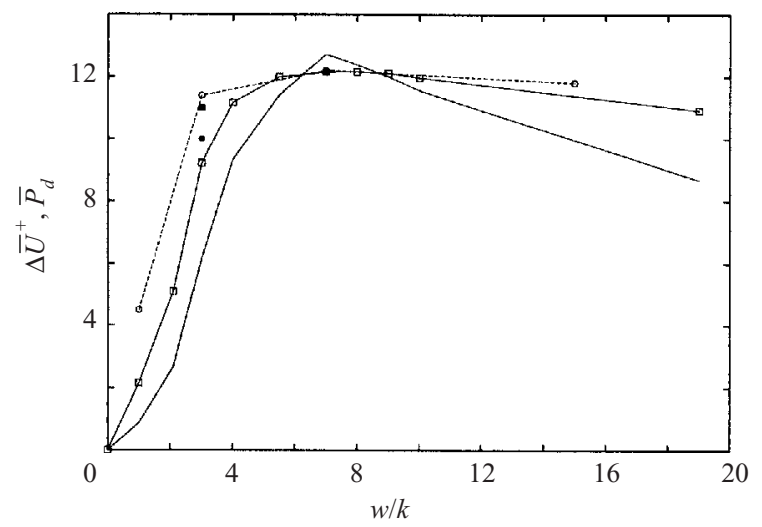

Figure 9. Comparison between the roughness function $\Delta \bar{U}^{+}$and the form $\operatorname{drag} \overline{P_{d}}$, each plotted against $w / k . \square$, present $\Delta \bar{U}^{+} ;------$, Furuya et al. (1976) transverse wires; Krogstad \& Antonia (1999) rods; $\bullet$, Moore (1951) square ribs;,$- \overline{P_{d}} \times 1000$.

shift occurs at $w / k=7$, i.e. the geometry which yielded the maximum value of $\overline{P_{d}}$. The resulting value of $\kappa$ varies significantly with $w / k$, ranging between 0.33 and 0.47 , so that the validity of (1.1) is questionable at least for the present Reynolds numbers. When both $U_{\tau}$ and $d_{o}$ are unknown, fitting to (1.1) could result in an incorrect estimation of $U_{\tau}$. With $\kappa$ taken as 0.41 , and by fitting the velocity profiles to $\bar{U}^{+}=(1 / 0.41) \ln y^{+}+C^{\prime}$, the magnitude of $C^{\prime}$ was estimated to be $2.9,-4,-6.2,-7.7$, $-7.6,-6.1$ for $w / k=1,3,4,7,9,19$ respectively. The corresponding distributions are shown as straight lines in figure $8(b)$. They compare favourably with the profiles obtained when the method of Jackson is used to determine $d_{o}$. The present results suggest that the roughness function, $\Delta \bar{U}^{+}$, depends only weakly on $d_{o}$. Figure 9 shows that $\Delta \bar{U}^{+}=C-C^{\prime}$ (with $C=5.5$ ) increases rapidly over the range $2<w / k<4$. For $4<w / k<10$, it is approximately constant, with a weak maximum at $w / k=7$. The distribution of total $\mathrm{drag} \overline{P_{d}}+\overline{C_{f}}$, shown in figure $5(a)$ and reproduced in figure 9 , is very similar to that for $\Delta \bar{U}$ and close to $\Delta \bar{U}^{+}$. The implication is that the pressure distribution around the roughness element influences $\Delta \bar{U}$ directly. When the flow reattaches on the bottom wall, $\Delta \bar{U}$ can be thought of as a momentum defect induced by the elements, and is therefore proportional to the drag (mostly form drag). Since $\Delta \bar{U}^{+}$is the ratio $\Delta \bar{U} / U_{\tau}$ and $U_{\tau}$ is also proportional to the drag, $\Delta \bar{U}^{+}$ is nearly constant. Over this range of $w / k$, there is reasonable agreement between the present distributions and the experimental data (results from three different experiments are included in figure 9). In particular, the present results confirm and extend the trend indicated by the wire roughness data obtained by Furuya et al. (1976). Larger differences (figure 9) between the present values of $\Delta \bar{U}^{+}$and those of Furuya et al. (1976) are found for small $w / k$ because the geometry differs in the two cases. For example, $w / k=0$ corresponds to a smooth wall in the present context, whilst in the Furuya et al. (1976) experiment, the wires touch each other, thus forming an undulating boundary.

\section{Conclusions}

The present study demonstrates the advantage of direct numerical simulations for providing reliable information on the pressure distributions around roughness 
elements and hence the form drag. The skin frictional drag $\overline{C_{f}}$ and the form $\operatorname{drag} \overline{P_{d}}$, which are extremely difficult to obtain in physical experiments, have been determined for a range of $w / k$, as well as several values of $k / h$. The distributions of $\left\langle C_{f}\right\rangle$ and $\langle P\rangle$, in conjunction with the mean streamlines, indicate that, for $w / k>7$, the roughness elements are isolated since the strength and size of the main recirculation zone no longer depend on $w / k$. The minimum $\overline{C_{f}}$ and maximum $\overline{P_{d}}$ occur at $w / k=7$ when the reattachment on the bottom wall occurs immediately upstream of the subsequent element. For $w / k \leqslant 2$, the total drag is closely approximated by the skin frictional drag on the crests of the elements. In the range $5<w / k<19$, the total drag is contributed almost entirely by $\overline{P_{d}}$. One would expect that, for this range, the Reynolds number dependence would be much smaller than in the previous case. The behaviour of $\Delta \bar{U}^{+}$, as a function of $w / k$, compares favourably with that previously obtained by experiment (Furuya et al. 1976) and reflects the way the form drag varies with $w / k$.

We acknowledge the support of the Australian Research Council, and the Ministero dell' Istruzione, dell' Università e della Ricerca and Centro di Eccellenza di Meccanica Computazionale, Politecnico di Bari.

\section{REFERENCES}

BANDYOPADHYAY, P. R. 1987 Rough-wall turbulent boundary layers in the transition regime. J. Fluid Mech. 180, 231-266.

Clauser, F. H. 1954 Turbulent boundary layers in adverse pressure gradients. J. Aero. Sci. 21, 91-109.

Fadlun, E. A., Verzicco, R., Orlandi, P. \& Mohd-Yusof, J. 2000 Combined immersed boundary finite-difference methods for three-dimensional complex flow simulations. J. Comput. Phys. 161, 35-60.

Furuya, Y., Miyata, M. \& Fujita, H. 1976 Turbulent boundary layer and flow resistance on plates roughened by wires. Trans. ASME: J. Fluids Engng 98, 635-644.

JACKSON, P. S. 1981 On the displacement height in the logarithmic profile. J. Fluid Mech. 111, 15-25.

Kim, J., Moin, P. \& Moser, R. 1987 Turbulence statistics in fully developed channel flow at low Reynolds number. J. Fluid Mech. 177, 133-166.

Krogstad, P.-Å. \& Antonia, R. A. 1999 Surface roughness effects in turbulent boundary layers. Exps. Fluids 27, 450-460.

LE, H., MoIn, P. \& KIM, J. 1997 Direct numerical simulation of turbulent flow over a backward-facing step. J. Fluid Mech. 330, 349-374.

LiU, C. K., Kline, S. J. \& Johnston, J. P. 1966 An experimental study of turbulent boundary layers on rough walls. Rep. MD-15, Department of Mechanical Engineering, Stanford University.

Moore, W. L. 1951 An experimental investigation of boundary layer development along a rough surface. PhD thesis, State University of Iowa.

NikURAdSE, J. 1933 Forschungshelft, no. 361 (Engl. transl. NACA TM 1292).

OrLandi, P. 2000 Fluid Flow Phenomena: A Numerical Toolkit. Kluwer.

Perry, A. E. \& Joubert, P. N. 1963 Rough wall boundary layers in adverse pressure gradients. J. Fluid Mech. 17, 193-211.

Perry, A. E., Schofield, W. H. \& Joubert, P. N. 1969 Rough wall turbulent boundary layers. J. Fluid Mech. 37, 383-413.

Raupach, M. R., Antonia R. A. \& Rajagopalan S. 1991 Rough-wall turbulent boundary layers. Appl. Mech. Rev. 44, 1-25. 